\title{
Magnetic characterization of the nickel layer protecting the copper wires in harsh applications
}

\author{
Daniel Roger, Stephane Duchesne, VAdim Iosif \\ Universitet Artois \\ daniel.roger@univ-artois.fr
}

(Received: 26.09.2016, revised: 26.11.2016)

\begin{abstract}
High Temperature $\left(\mathrm{HT}^{\circ}\right)$ motor coils open new perspectives for extending the applications of electrical motors or generators to very harsh environments or for designing very high power density machines working with high internal temperature gradients. Over a temperature of $300^{\circ} \mathrm{C}$, the classic enameled wire cannot work permanently, the turn-to-turn insulation must be inorganic and made with high temperature textiles or vitro-ceramic compounds. For both cases, a diffusion barrier must protect the copper wire against oxidation. The usual solution consists of adding a nickel layer that yields an excellent chemical protection. Unfortunately, the nickel has ferromagnetic properties that change a lot the skin effect in the HT wire at high frequencies. For many applications such as aeronautics, electrical machines are always associated with PWM inverters for their control. The windings must resist to high voltage short spikes caused by the fast fronted pulses imposed by the feeding inverter. The nickel protection layer of the $\mathrm{HT}^{\circ}$ inorganic wire has a large influence on the high frequency behavior of coils and, consequently, on the magnitude of the voltage spikes. A good knowledge of the non-linear magnetic characteristics of this nickel layer is helpful for designing reliable HT inorganic coils. The paper presents a method able to characterize non-linear electromagnetic properties of this nickel layer up to $500^{\circ} \mathrm{C}$.
\end{abstract}

Key words: high temperature motor winding, nickel diffusion barrier, temperature dependent magnetic properties

\section{Introduction}

Electrical machines able to work at high temperatures $\left(300-400^{\circ} \mathrm{C}\right)$ for short times are now standard products; they are used for smoke extractors. These machines are built with an improved Electrical Insulation System (EIS) able to withstand high temperatures during short lifespans. They use high performance polymers such as polyimide (PI) strengthened by inorganic particles [1]. However, these conventional organic technologies reach their limits, continuous operations at such high temperature are not possible.

Electrical machines able to operate continuously at $400^{\circ} \mathrm{C}$ have been developed by the petroleum industry for deep drilling [2]. Their EIS are built with composite materials that 
combine fiberglass and mica. The large thicknesses of these inorganic insulating layers $(100-200 \mu \mathrm{m})$ limits this inorganic technology to big machines using large wire sections.

Many applications, in ground transports or in aeronautics require compact actuators of low and medium powers. With higher permanent internal temperatures, it is possible to design machines with higher current densities and consequently higher power densities. The coils of such machines are built with a wire insulated by a thin inorganic layer made of a glass-ceramic compound. Unfortunately, this inorganic insulating layer is porous; it is no longer able to protect the copper against oxidation. Therefore, the copper must have an additional protection layer made of nickel, between the copper and the inorganic insulation layer. The copper wire can be used up to $230^{\circ} \mathrm{C}$ without any protection and with a porous inorganic insulation. A nickel protection layer must be added for higher temperatures. Manufacturers propose wires with a higher nickel thicknesses for the products able to operate at the most elevated temperatures [3].

Nickel is a ferromagnetic material, therefore the protection layer changes the electromagnetic global behavior of the coil, especially in the higher part of the frequency spectrum because of skin and proximity effects. The paper presents an experimental method able to characterize the non-linear magnetic properties of the nickel protection layer of $\mathrm{HT}^{\circ}$ inorganic wires, it gives details on the research works presented in the conference EPNC 2016 [4].

\section{High temperature coils}

Fig. 1 is the picture of such a motor coil after tests at $500^{\circ} \mathrm{C}$. This rigid coil is made with an available inorganic $\mathrm{HT}^{\circ}$ wire [5] protected by a high temperature cement based on alumina [6]. These coils are placed on the stator teeth of concentrated winding permanent magnet synchronous machines (CW-PMSM) [7].

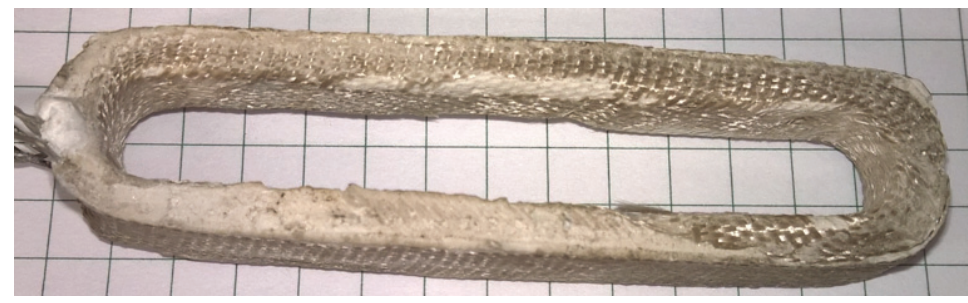

Fig. $1 . \mathrm{HT}^{\circ}$ inorganic coil after tests at $500^{\circ} \mathrm{C}$

Fig. 2 is a microscopy of the cross section of a $\mathrm{HT}^{\circ}$ inorganic wire, which diameter is $800 \mu \mathrm{m}$. The nickel layer thickness is $65 \mu \mathrm{m}$ and the inorganic insulation one $10 \mu \mathrm{m}$. For modeling the high frequency behavior of $\mathrm{HT}^{\circ}$ coils made with such a wire, the magnetic permeability of the nickel, for several temperature, is required. A study made a long time ago shows a strong dependence of the permeability to the magnetic field and the temperature [8]. Deeper investigations are useful for considering the non-linear phenomena of this nickel layer. 


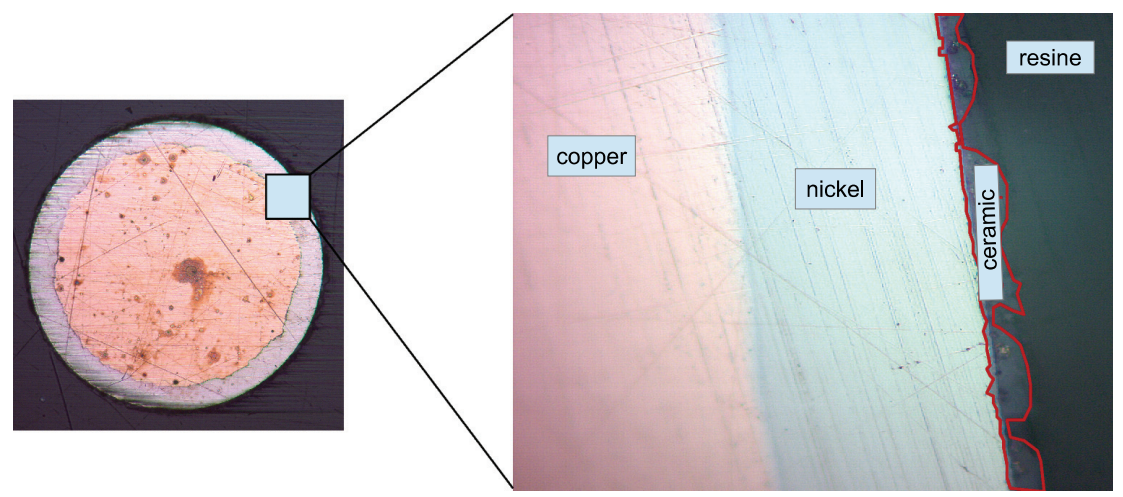

Fig. 2. $\mathrm{HT}^{\circ}$ inorganic wire cross-section

\section{Measurements at low temperatures}

The experimental setup defined for measuring the $B(H)$ curve of the nickel layer is inspired from the Epstein frame. Measurements are performed at low frequency, the skin effects is negligible. The $\mathrm{HT}^{\circ}$ inorganic wire is wound to form the magnetic core of the experimental system presented in Fig. 3. The two primary coils connected in series produce the magnetic field and the secondary coils measure the global magnetic flux. Fig. 4 gives the electric circuit for measurements.

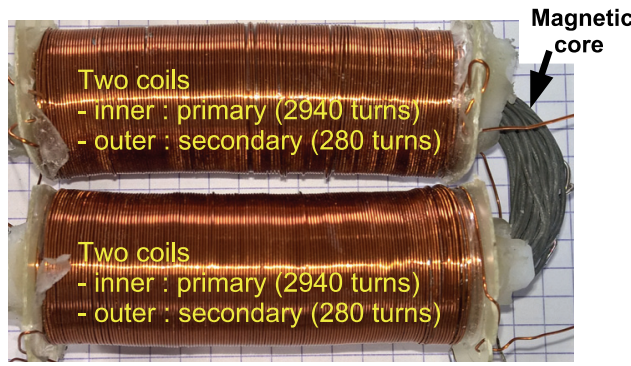

Fig. 3. Measurement device at ambient temperature

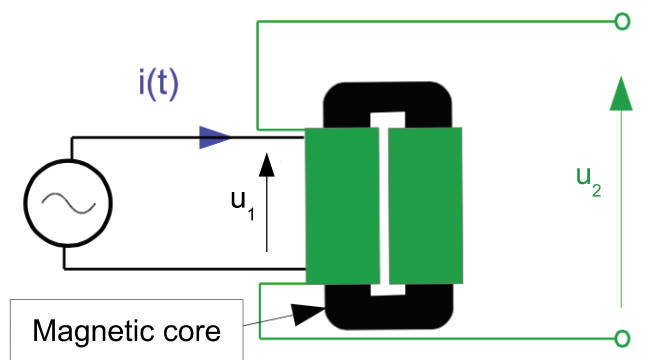

Fig. 4. Electric circuit for measurements at ambient temperature 
In the magnetic core the nickel clad wire follows the field lines created by the primary coils. Therefore, the magnetic field is tangent to the nickel layer at any point of the wire periphery as shown in Fig. 5. With this geometry, the principle of conservation of the tangential component of the field on each side of the border between the materials impose the equality of the magnetic field in the air, the nickel and the copper.

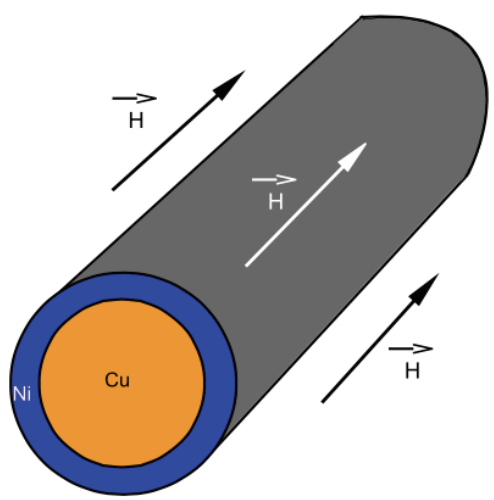

Fig. 5. Detail of the magnetic field in the experimental core

The magnitude of this field can be estimated by the Ampere law from the primary current $i$, the turn number of the primary winding $N_{1}$ and the average length of the experimental core $l$.

$$
H=\frac{N_{1} i}{l} .
$$

The Faraday law yields the voltage induced in the secondary from the total flux $\varphi$ and the number of secondary turns $N_{2}$.

$$
u_{2}=N_{2} \frac{\mathrm{d} \varphi}{\mathrm{d} t} .
$$

The flux is computed by integrating the secondary voltage. The results are presented in Fig. 6 for $f=50 \mathrm{~Hz}$. The straight-line gives the flux computed considering only the uniform flux in the air inside a virtual tube following the core and of the same diameter as the seconddary coils. The non-linearity caused by the nickel layer is clearly visible.

The flux density in the nickel layer is computed from the flux measurements by subtracting the flux in the tube of air and dividing by the nickel section. The resulting hysteresis loop is presented in Fig. 7.

The hysteresis loop shows the ferromagnetic properties of the nickel layer with a saturation shoulder at approximately $B_{S}=0.35 \mathrm{~T}$ for a field $H_{S}=4000 \mathrm{~A} / \mathrm{m}$. The width of the loop is quite large but the eddy currents induced in the copper central wire artificially increase this value. The key information from this experience is an estimation of the current that corresponds to the saturation shoulder of the nickel layer. Considering a single conductor, which radius is $a$, away from any external electromagnetic influence, the current corresponding to the 
saturation shoulder is that corresponds to a current of $10 \mathrm{~A}$ peak, for a wire of diameter $0.8 \mathrm{~mm}$. The corresponding average current density is $19.9 \mathrm{~A} / \mathrm{mm}^{2}$, which is very high, even for a HT motor.

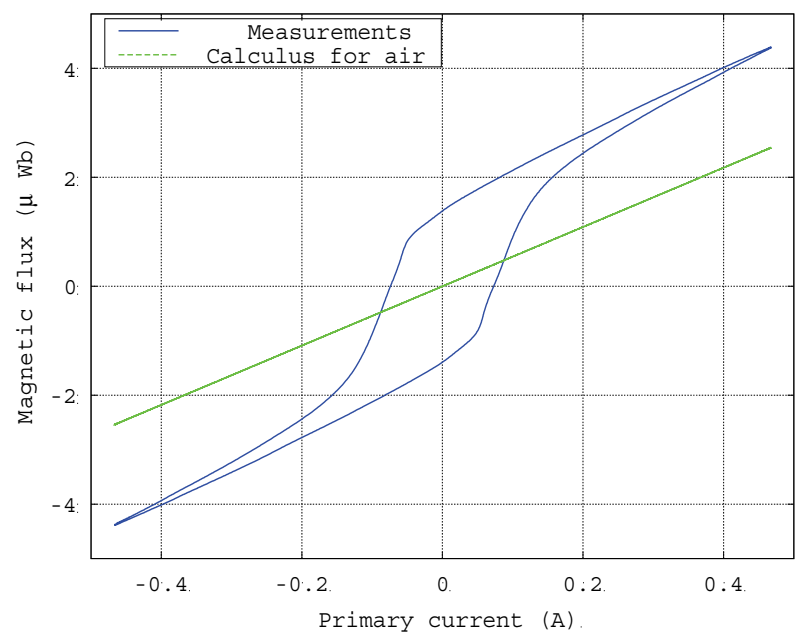

Fig. 6. Flux in the core as a function of the primary current

The hysteresis loop is very similar to that for iron, but with a saturation shoulder at a lower level. Consequently, the relative permeability is very difficult to define because of the nonlinear behavior of this ferromagnetic material, it depends on minor hysteresis loops, dc magnetic polarization and ac field magnitude.

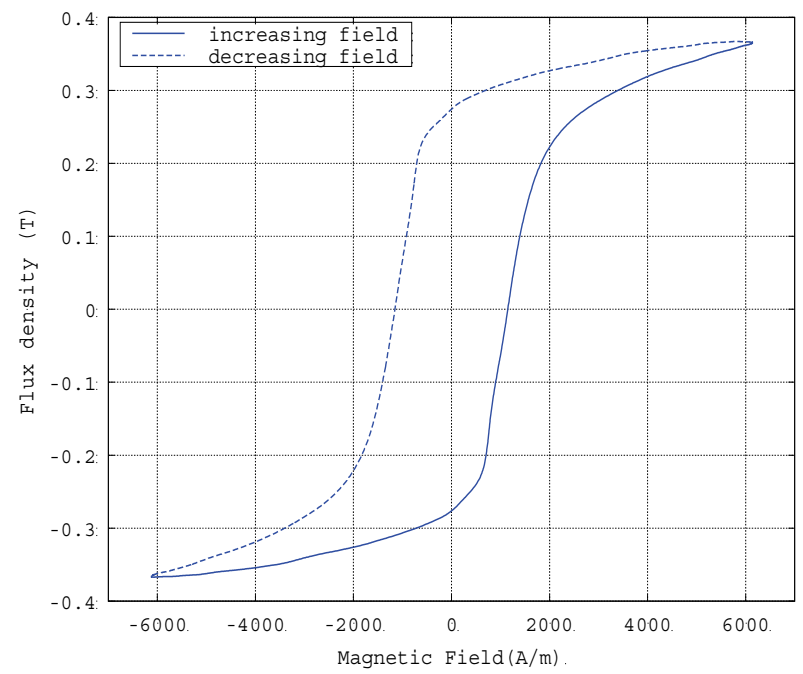

Fig. 7. Hysteresis loop of the nickel protection layer 


\section{Investigations at high temperatures}

For testing the influence of the nickel layer at high temperatures, a simpler experimental setup has been made, it consists of two adjacent $\mathrm{HT}^{\circ}$ wires attached to a mica plate by spots of HT $^{\circ}$ cement. Fig. 8 shows a small part of the experimental bifilar line, which total length is $3 \mathrm{~m}$.

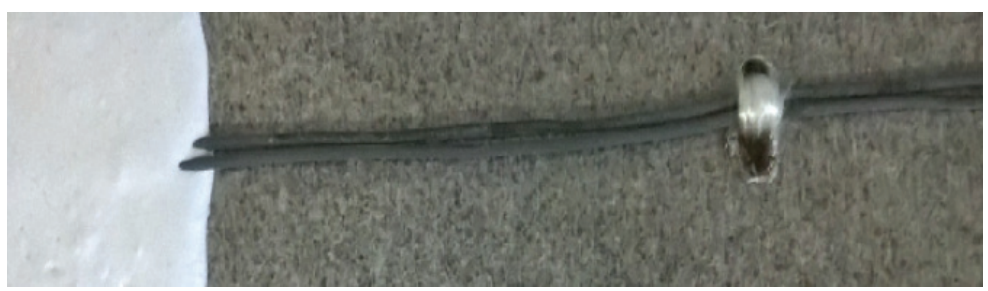

Fig. 8. Small part of the $3 \mathrm{~m}$ length bifilar line set on a mica plate

The end of the pair is short-circuited and its input is connected to the measurement device by a much shorter twisted pair made with mica fiberglass $\mathrm{HT}^{\circ}$ wire. The inductance measurements are used for estimating the nickel layer properties. Fig. 9 presents the inductance measurements performed on the bifilar experimental line versus temperature for two currents imposed by the measurement system. The low current imposed by the impedance analyzer corresponds to a low field between the two wires yields lower inductance that measurements performed at higher currents.

The measurements show a strong dependence of the inductance to the temperature and the magnetic field due to the nickel protection layer.

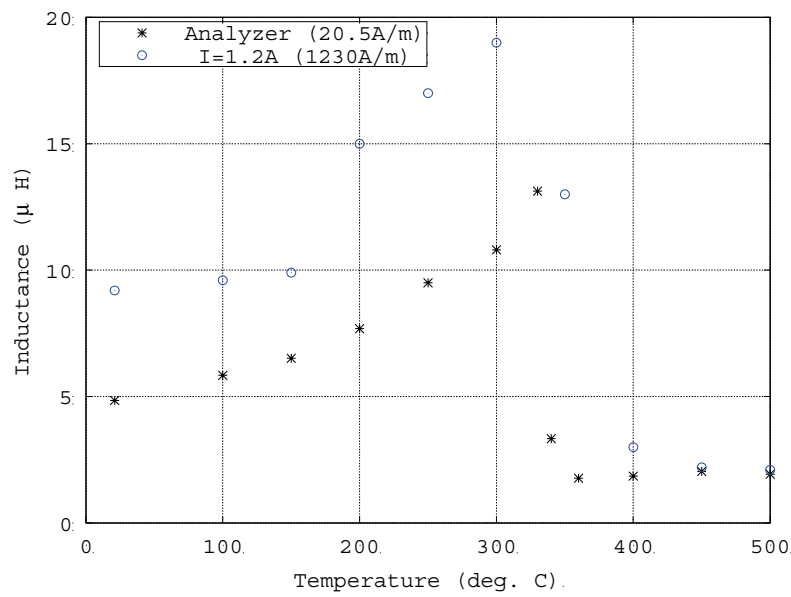

Fig. 9. Temperature dependence of the experimental bifilar line inductance

The nickel layer permeability at any temperature can be deduced from the bifilar line inductance measurement. Unfortunately, the actual distance between the wires of the experimen- 
tal bifilar line is not known with a relevant accuracy as it can be seen on Fig. 8. Therefore, the average value of this parameter is computed from electrical measurements performed above the Curie point, when the ferromagnetic properties of the nickel layer are nullified. For these temperatures and for low frequencies, the bifilar line inductance can be estimated using the classical analytic approach that consider homogeneous wires, which geometric parameters are defines in Fig. 10.

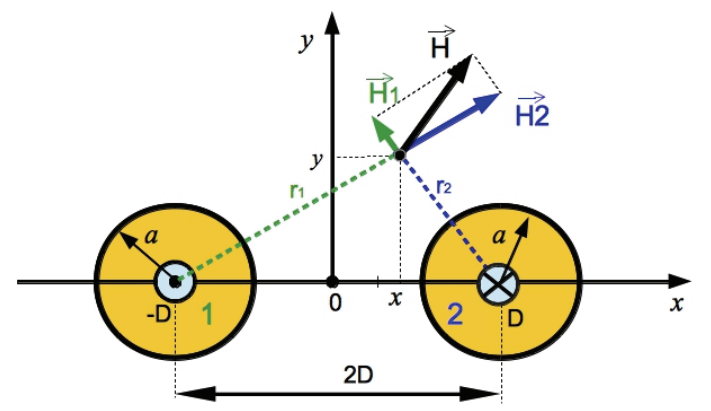

Fig. 10. Cross-section of the bifilar line and geometric parameters

The magnetic field is computed applying the Ampère's law and considering currents in opposite direction in the two wires. The flux is obtained by performing the integration along the $x$ axis between the points corresponding the null value of the flux density. Then, the inductance $L$, for a length $l$ of the bifilar line, is obtained dividing the flux by the current. Considering the geometric parameters defined in Fig. 10, the global result is:

$$
\frac{L}{1}=\frac{\mu_{0}}{\pi}\left(\operatorname{Ln} \frac{D+\sqrt{D^{2}+a^{2}}}{a}+\frac{1}{a^{2}}\left(D \sqrt{D^{2}+a^{2}}\right)\right) .
$$

This analytical approach is based on 2 wires made of homogeneous materials, but the real wire over the Curie point is not homogeneous because the resistivity of the nickel is much higher than the copper one $\left(26.10^{-8} \Omega \cdot \mathrm{m}\right.$ and $4.19 .10^{-8} \Omega \cdot \mathrm{m}$ respectively at $\left.400^{\circ} \mathrm{C}\right)$. A Finite Element (FE) simulation has been made for observing the differences between the analytical approach and the reality. Fig. 11 highlights this approximation showing the fields on the $x$ axis.

The difference between the curves $H$ and $E F$ denotes an under-evaluation of the flux therefore of the inductance. A lower error of the analytical model is obtained choosing $a=r_{\mathrm{Cu}}$, the radius or the copper core of the wire. Fig. 12, drawn with this hypothesis, shows the lower under-evaluation for the improved model.

The parameter $\mathrm{D}$, corresponding to the half of the average distance between the center of the wires of the experimental bifilar line is determined from the analytical formulae (3) of the improved model where $a=r_{\mathrm{Cu}}$. The input data are: the inductance measured at $400^{\circ} \mathrm{C}$ $(L=1.94 \mu \mathrm{H})$; the radius of the copper core of the wire $\left(a=r_{\mathrm{Cu}}=325 \mu \mathrm{m}\right)$ and the length of the line $(l=3 \mathrm{~m}) . D$ is the only unknown parameter of Eq. (3) that can be computed by a numerical method. The result is $D=485 \mu \mathrm{m}$. 


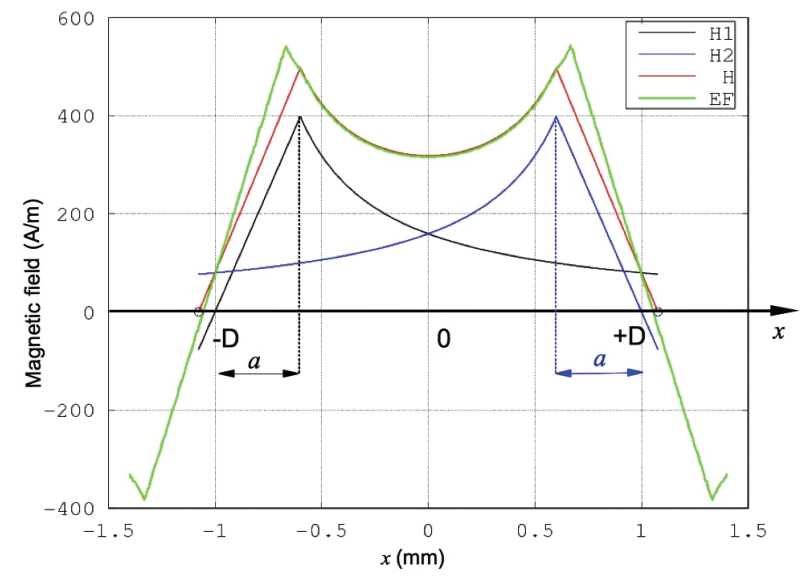

Fig. 11. Magnetic field on the $x$ axis (H1: left wire, $H 2$ : right wire, $H$ : resulting field, $E F$ : field computed taking the higher resistivity of the nickel at $400^{\circ} \mathrm{C}$ into account

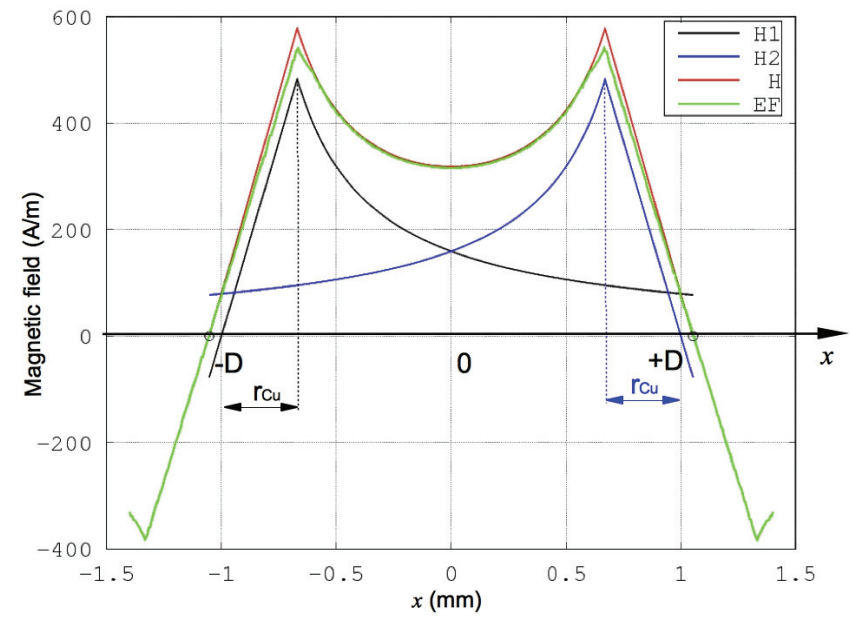

Fig. 12. Improved analytical model obtained with $a=r_{\mathrm{Cu}}$ in the same conditions

With an accurate determination of the average distance $2 D$ between the wire center of the experimental line and the inductance measurements at temperature under the Curie point, it is possible to determine the magnetic permeability of the nickel layer at any temperature.

The flux density is $\mu_{0} \mathrm{H}$ in the copper and in the air and $\mu_{0} \mu_{R} \mathrm{H}$ in the nickel layer as illustrated in Fig. 13. The flux increase $\Delta \phi$ due to the relative permeability $\mu_{R}$ of the nickel layer is due to its ferromagnetic properties under the Curie point and corresponds to the hatched areas in Fig. 13. This increase, for a length $l$ of the bifilar line and nickel thickness $e_{n}$ is:

$$
\frac{\Delta \phi}{1}=2\left(B_{2}-B_{1}\right) e_{n}
$$




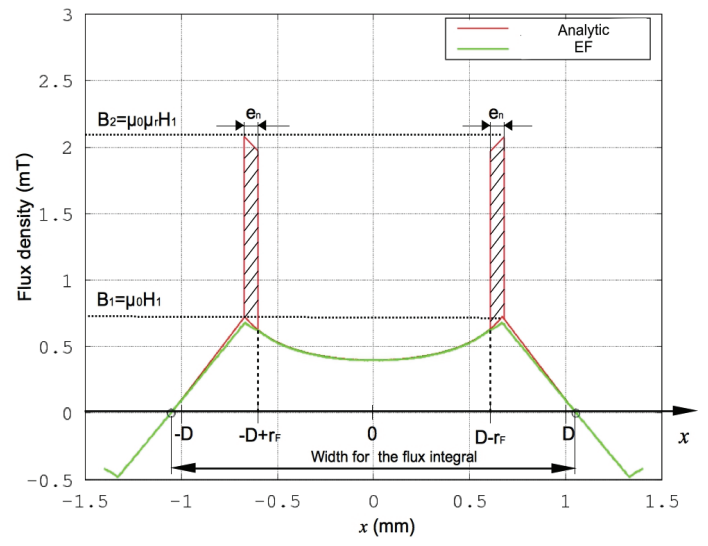

Fig. 13. Increase of the flux density in the nickel layers

The improved analytical model gives the maximum value $H_{1}$ of the magnetic field; therefore the increase of the inductance $\Delta L$, comparing to the value measured over the Curie point, can be used for determining the relative permeability of the nickel at any temperature and current measurement. The final formulae is given by Eq. (5) were $e_{N}$ is the nickel thickness and $r_{F}$ the total wire radius with the nickel layer.

$$
\mu_{R}=1+\frac{\Delta L}{1} \frac{\pi}{\mu_{0}\left[\frac{1}{2 D-\left(r_{F}-e_{n}\right)}+\frac{1}{r_{F}-e_{n}}\right]} .
$$

The application of this equation to the inductance measurements performed up to $400^{\circ} \mathrm{C}$ are presented in Fig. 14.

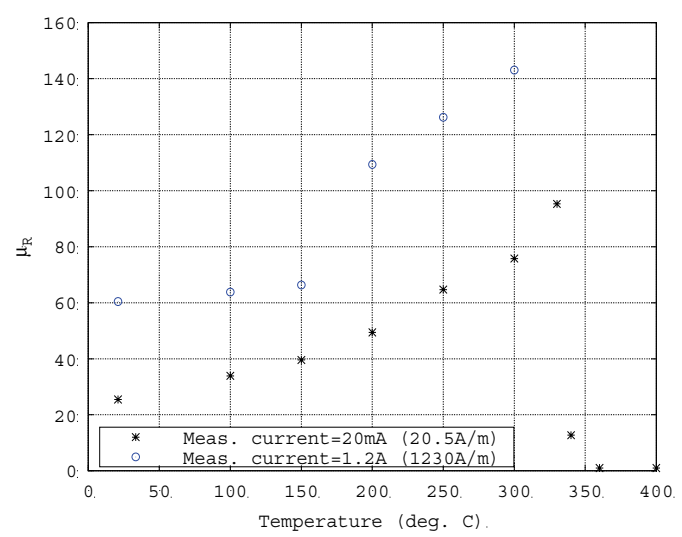

Fig. 14. Relative permeability of the nickel layer versus temperatures at two current levels

There is a significant increase of the magnetic permeability below the Curie point $\left(360^{\circ} \mathrm{C}\right)$ and a brutal decrease over the Curie point as expected for a ferromagnetic material [9]. These 
results show that the relative permeability is also strongly dependent of the ambient field in the neighborhood of the nickel layer.

\section{Conclusions}

The proposed experimental approach describes a method able to characterize the nonlinear magnetic characteristics of the nickel layer used as a diffusion barrier in $\mathrm{HT}^{\circ}$ wires used in a harsh environment. The proposed method is based on the analysis of the behavior of a bifilar short-circuited line placed in an oven.

The preliminary measurements made at ambient temperature show the hysteresis loop of the thin nickel layer and a saturation shoulder at more or less $0.3 \mathrm{~T}$. This hysteresis loop, which is similar to the one measured on classic iron, predicts a very non-linear behavior. Consequently, the high frequency model of the motor coils used for predicting the voltage spikes during transients will be more complex. Such a model is all the more useful as the electrical performances of the $\mathrm{HT}^{\circ}$ inorganic insulating materials are poor compared to those of conventional polymers [10]. A fine modeling of the high frequency electromagnetic behavior of the coils is crucial for designing $\mathrm{HT}^{\circ}$ machines powered by PWM inverters, which impose very stiff and repetitive voltage edges [11].

For temperature below the Curie point of nickel, the nickel diffusion barrier modifies strongly the skin and proximity effects: the higher damping effects mitigate the voltage spikes after each voltage edge. Consequently, the dielectric stress on the turn-to-turn insulation is lower. A good knowledge of the non-linear magnetic characteristics of the diffusion barrier added to the copper wire is helpful for designing reliable $\mathrm{HT}^{\circ}$ inorganic machines that will be able to withstand the fast fronted voltages imposed by the PWM inverter that feed the machine. When this $\mathrm{HT}^{\circ}$ winding technology is mature, it will be possible to design large power generators working in the near vicinity or the propulsion turbines of aircrafts.

\section{Acknowledgements}

This work is a part of the project "Compact Electric Actuator with Integrated Converter for Extreme temperatures - ACCITE" supported by the French Foundation for Aeronautics and Espace (FRAE).

\section{References}

[1] Aymonino F., Lebey T., Malec D., Petit C., Michel J., Anton A., Gimenez A., Degradation and dielectrics measurements of rotating machines insulation at high temperature $\left(200-400{ }^{\circ} \mathrm{C}\right)$, IEEE International Conference on Solid Dielectrics, July 2007, pp. 130-133 (2007).

[2] Hooker M., Hazelton C., Kano K., Tupper M., High-temperature electrical insulation for EGS downhole equipment, Thirty-Fifth Workshop on Geothermal Reservoir Engineering, Stanford University, Stanford, California (2010).

[3] Vonroll, Mica-taped wires samicafirewall, www.vonroll.com (2015). 
[4] Iosif V., Roger D., Duchesne S., Magnetic characterization of the nickel protection layer of wires used for high temperature motors, XXIV Symposium on Electromagnetic Phenomenon and Nonlinear Circuits (EPNC), Helsinki (2016).

[5] Plastec Group, Cerafil500. www.cables-cgp.com/.

[6] Cotronics, Adhesifs, ciments, revetements ceramiques hautes temperatures -1000 à $3000^{\circ} \mathrm{C}$, www.cotronics.com/.

[7] Iosif V., Roger D., Takorabet N., Duchesne S., Meibody-Tabar F., Technological assessments for designing machines able to work at very high internal temperatures $\left(450-500{ }^{\circ} \mathrm{C}\right)$, International Conference on Electrical Machines (ICEM), Lausanne (2016).

[8] Zaimovski A., On the temperature dependence of magnetic permeability in weak fields, Journal of Physics (1941).

[9] Néel L., Propriétés magnétiques du nickel pur a proximité du point de curie, Société Française de physique (1933).

[10] Iosif V., Roger D., Duchesne S., Malec D., Assessment and improvements of inorganic insulation for high temperature low voltage motors, IEEE Transactions on Dielectrics and Electrical Insulation, vol. 23, no. 5, pp. 2534-2542 (2016).

[11] Iosif V., Duchesne S., Roger D., Voltage stress predetermination for long-life designof windings for electric actuators in aircrafts, IEEE - Annual Report Conference on Electrical Insulation and Dielectric Phenomena (CEIDP), Ann Arbor, pp. 319-321 (2015). 